Rev. Elev. Méd. vét. Pays trop., 1972, 25 (2): 187-189

\title{
Note sur le rôle vecteur des rapaces dans la propagation de certaines maladies bactériennes
}

\author{
par J. BLANCOU (*) et J. RAJAONARISON (*)
}

\begin{abstract}
RESUME
Des rapaces nourris de cadavres d'animaux morts de charbon bactéridien, charbon symptomatique ou colibacillose éliminent les bactéries correspondantes durant 4 jours au moins dans leurs excréments. Ce délas est suffisant à une espèce migratrice pour parcourir plus de $2.500 \mathrm{~km}$ : l'hypothèse de la possibilité de la propagation de ces matadies par les migrations aviennes est donc démontrée, spécialement d'Afrique à Madagascar.
\end{abstract}

\section{INTRODUCTION}

Le rôle des animaux sauvages dans la diffusion des maladies a été suspecté ou démontré très souvent. Une revue complète de cette question a été faite en 1962 par A. Mac DIARMID (6), puis M.A. LOBRY (5) en Afrique.

Cependant une des inconnues majeures signalées dans ces revues est le rôle possible des oiseaux migrateurs dans le transport d'agents pathogènes pour l'homme ou l'animal. Lorsqu'on sait les trajets empruntés par ces oiseaux, trajets qui peuvent atteindre $12.000 \mathrm{ki}-$ lomètres (4), on est tenté d'établir une relation entre l'apparition des maladies dans un pays et l'arrivée de ces grands migrateurs. Cette hypothèse a donc été émise à plusieurs reprises. et en particulier à Madagascar $(7,1,2)$ dont l'insularité n'est pas toujours une garantie certaine contre les épizooties africaines.

Nous avons tenté, dans la présente étude, de contrôler ces suppositions. Pour cela nous nous sommes efforcés de vérifier en laboratoire l'hypothèse d'un rapace se nourrissant d'un

(*) I.E.M.V.T., Laboratoire régional, B.P. n" 862 , Tananarive, Madagascar. cadavre infectant en Afrique et venant souiller de ses déjections le cadavre d'un autre animal de Madagascar.

Plusieurs espèces aviennes migrent en effet d'Afrique à Madagascar (3) dont au moins un rapace nécrophage Milvus migrans migrans (BODDAERT). La durée de la traversée du Canal de Mozambique ne doit pas excéder une matinée, à la vitesse moyenne du vol de ces oiseaux qui est de 50 kilomètres à l'heure.

\section{Rapace}

\section{MATERIEL}

La buse malgache "Buteo brachypterus" (HARTLAUB), nécrophage à l'occasion, a été choisie pour sa commodité de manipulation.

\section{Bactéries}

Les souches des germes responsables des trois maladies épizootiques bovines existant actuellement dans l'Ile (charbon bactéridien, charbon symptomatique et entérite colibacillaire) utilisées ont été les suivantes :

Bacillus anthracis: souche " A 43 " du laboratoire, mortelle pour le cobaye à la dose de $0,1 \mathrm{ml}$ de culture en bouillon ordinaire; 
Clostridium chauvei: souche « $735 »$ de laboratoire, mortelle pour le cobaye à la dose de $0,125 \mathrm{ml}$;

Escherichia coli: souche $111 \mathrm{~B} 4$, mortelle pour le cobaye à la dose de $0,5 \mathrm{~mL}$.

\section{METHODES}

De nombreuses expériences successives ont été réalisées avec ces trois souches. Dans chacune d'elles le régime alimentaire normal de l'oiseau (viande crue) était interrompu par un unique repas infectant constitué d'un mélange de viscères sanglants (foie, rate, reins) d'un cobaye inoculé la veille par la bactérie étudiée. Cette bactérie était alors recherchée quotidiennement dans les excréments de l'oiseau, isolée et identifiée selon les méthodes suivantes :

Bacillus anthracis: la seule méthode satisfaisante, compte tenu de l'importante contamination des fèces, est l'isolement sur gélose nutritive dune suspension de fèces chauffée 5 minutes à $80^{\circ}$. Les colonies ayant une morphologie de Bacillus et contenant des bactéries immobiles sont réisolées et jdentifiées par leurs caractères biochimiques et pathogènes.

Clostridium chauvei: les fèces sont diluées au $1 / 10$ en eau physiologique et inoculées par voie intramusculaire au cobaye sous le volume de $1 \mathrm{ml}$. Comme ces fèces contiennent toujours un nombre élevé de Welchia perfringens (plus de 100 par g) l'inoculation doit se faire sous couvert d'une séro-protection, par 1.350 unités de sérum anti-Welchia perfringens type $\mathrm{A}$, assurée 4 à 8 heures auparavant.

Lörsque le cobaye meurt avec les lésions caractéristiques du charbon symptomatique, une hémoculture est réalisée. Le Clostridium y est recherché et identifié par ses caractères culturaux, biochimiques, pathogènes et sérologiques (survie de cobayes séro-protégés par 3.000 unités de sérum anti-Clostridium chauvei, et mort des témoins).

Escherichia coli: les fèces sont ensemencées directement sur gélose "S.S. » et les colonies fermentant le lactose en sont réisolées. Leur agglutination est alors recherchée dans le sérum monospécifique $111 \mathrm{~B} 4$.

\section{RESULTATS}

Ils sont rapportés dans le tableau ci-dessous, où ne figurent que les records de persistance des trois espèces bactériennes. Ni avant le repas infectant, ni au-delà des temps indiqués les bactéries n'ont pu être réisolées des fèces.

TABLEAU $\mathrm{N}^{\circ} \mathrm{I}$

\begin{tabular}{|l|c|c|c|c|c|c|}
\hline \multirow{2}{*}{$\begin{array}{c}\text { Espèce } \\
\text { bactérienne }\end{array}$} & \multicolumn{5}{|c|}{ Isolement de la bactérie dans les fèces après : } \\
\cline { 2 - 7 } & $24 \mathrm{~h}$ & $48 \mathrm{~h}$ & $72 \mathrm{~h}$ & $96 \mathrm{~h}$ & $110 \mathrm{~h}$ & $134 \mathrm{~h}$ \\
\hline $\begin{array}{l}\text { Bacizius } \\
\text { anthracis }\end{array}$ & - & - & - & + & - & - \\
\hline $\begin{array}{l}\text { clostriaium } \\
\text { chawvei }\end{array}$ & + & - & + & + & - & - \\
\hline $\begin{array}{l}\text { Escheriohia } \\
\text { colii }\end{array}$ & + & - & - & + & - & - \\
\hline
\end{tabular}

\section{CONCLUSION - DISCUSSION}

Il convient de noter tout d'abord la remarquable résistance de Buteo brachypterus aux trois bactéries étudiées, bactéries ingérées à des doses le plus souvent mortelles pour des mammifères d'un poids correspondant.
La durée de conservation des trois espèces bactériennes étudiées peut donc atteindre 3 à 5 jours chez l'oiseau vecteur. Cette persistance de l'agent pathogène permet théoriquement à un rapace migrateur de transmettre une maladie contagieuse dont le foyer est situé à plus de $2.500 \mathrm{~km}$ de distance. Ces infections peuvent 
donc d'une façon très plausible franchir le canal de Mozambique; cette possibilité n'est réduite que par le jeu du hasard qui doit entraîner l'oiseau successivement sur deux cadavres animaux dans les trois jours qui précèdent ou suivent sa migration saisonnière.

\section{Remerciements}

Nous remercions vivement la Direction de l'ORSTOM à Tananarive et le Docteur R. ALBIGNAC qui nous ont aimablement fourni les rapaces utilisés dans ces expériences.

\section{SUMMARY}

Note about the role of birds of prey as vectors in the spreading of some bacterial diseases

Birds of prey fed with animals dead from anthrax, blackleg or colibacillosis reject correspondent bacteria at least 4 days in their excrements. This time is enough for migratory species to fly over more than 2.500 kilometers. So the possibility of an spreading of these disease by birds's migration is proved, particularly from Africa to Madagascar.

\section{RESUMEN}

\section{Nota sobre el papel vector de Ios rapaces en la propagación de ciertas enfermedades bacterianas}

Rapaces alimentados con cadaveres de animales muertos de carbunco bacteridiano, de carbunco sintomático o de colibacilosis eliminan las bacterias correspondientes durante 4 dias por lo menos en sus excrementos. Este duración es suficiente para que una especie de paso recorra más de 2.500 kilometros: Asi se demostra la hipotesis de la posibilidad de la propagación de dichas enfermedades por las migraciones de las aves, particularmente de Africa hacia Madagascar.

\section{BIBLIOGRAPHIE}

1. BLANCOU (J.), RAKOTOARIVELO (J.), SERRES (H.), Note sur les premiers cas de charbon symptomatique à Madagascar, Rev. Elev. Méd. vét. Pays trop., 1971, 24 (1) : 19-21.

2. BUCK (G.), QUESNEL (J. J.), SERRES (H.), Une maladie nouvellement identifiée à Madagascar: la "Lumpy skin disease ", Rev'. Elev'. Méd, vét. Pays" trop., 1956, 9 (3) : 229-35.

3. DELACOUR (J.), Liste systématique des oiseaux récoltés à Madagascar par la mission FrancoAnglo-Américaine, Oiseau, 1932, 2 : 1-96.
4. DORST (J.), Les migrations des oiseaux. Paris, Payot, 1950.

5. LOBRY (M. A.), Existence chez les animaux sauvages en Afrique de cas de maladies infectieuses des animaux domestiques, Bull. épiz. Dis. Afr., 1964, 12: 43-62.

6. Mac DIARMID (A.), Maladies des animaux sauvages vivant en liberté. Rome, F.A.O., 1962. (Etudes agricoles de la F.A.O.. no 57.)

7. Rapports annuels du Laboratoire central de l'élevage de Tananarive. Archives I E M.V.T., MaisonsAlfort (France). 